\title{
DENSIDADE DO FERRO BIODISPONIIVEL EM UMA DIETA HABITUAL NO ESTADO DE SÃO PAULO*
}

SZARFARC, S. C. Densidade do ferro biodisponivel em uma dieta habitual no Estado de São Paulo. Rev. Saúde públ., S. Paulo, 17:290-6, 1983.

RESUMO: O conhecimento do potencial de biodisponibilidade de ferro da dieta de uma população é imprescindivel na implantação de um programa de fortificação com esse mineral. A quantificação da capacidade de absorção do ferro dietético foi efetuada em três refeiçōes, habituais entre os paulistas: desjejum (pão, margarina, café com açúcar), almoço (arroz, feijão, carne e café com açúcar) e jantar (arroz, feijão, ovo frito, café com açúcar), servidas a 28 individuos adultos, de ambos os sexos, aparentemente sadios. As densidades do ferro biodisponível ( $\mathrm{dFeB}$ ) nas refeições estudadas foram: desjejum $\mathrm{dFeB}=0,23$; almoço $\mathrm{dFeB}=0,73$ e jantar $\mathrm{dFeB}=0,28$. Os valores encontrados, bastante inferiores aos adequados para suprir o requerimento de ferro da mulher, sugerem que a fortificação não é estratégia efetiva no controle da anemia ferropriva, quando aplicada às refeições de composição igual àquelas estudadas.

UNITERMOS: Ferro. Dieta. Inquéritos nutricionais, Estado de São Paulo, Brasil.

\section{INTRODUCCAO}

A baixa densidade de ferro*** da dieta consumida em relação à recomendada. especialmente entre mulheres e crianças, explica, em grande parte, a elevada prevalência com que a anemia ocorre nesses grupos populacionais.

$O$ fornecimento de ferro e de energia, pelos diversos grupos de alimentos, para a população do Estado de São Paulo ${ }^{3}$ (Tabela 1), mostra que o valor da densidade do ferro ***dFe $=6$, é comum a toda à população e satisfaz as necessidades dela como um todo ( $14 \mathrm{mg} \mathrm{Fe} / 2.400 \mathrm{kcal})$. Este valor porém, está longe de atingir o recomendado pelo National Research Council" para a mulher adulta, dFe $=8,6(18 \mathrm{mg} \mathrm{Fe} / 2.100$ kcal) e mais distante ainda do recomendado pela FAO e adaptado por Martinss para a mulher brasileira (28mg Fe $/ 2.150 \mathrm{kcal}$ ) $\mathrm{dFE}=13,3$.

A fortificação de alimentos com ferro é uma estratégia importante na modificação dessas distorçōes, permitindo alterar o teor do nutriente mantendo seu conteúdo energético.

O valor da densidade de ferro ${ }^{11}$, embora sendo indicador sensivel do mineral disponível por unidade energética, não pode ser utilizado na determinação da quantidade do

* Trabalho realizado em convênio com Instituto Venezolano de Investigaciones Cientificas/Universidade das Nações Unidas (IVIC/UNU).

* Do Departamento de Nutrição da Faculdade de Saúde Pública da Universidade de São Paulo - Av. Dr. Arnaldo, 715 - 01255 - São Paulo, SP - Brasil.

*** (mg de ferro da dieta/calorias da dieta) $\mathrm{x} 1.00011$. 
SZARFARC, S.C. Densidade do ferro biodisponível em uma dieta habitual no Estado de São Paulo. Rev. Saúde pübl., S. Paulo, 17:290-6, 1983.

T A B E L A 1

Consumo de energia e ferro, per capita, diário, e densidade de ferro, segundo grupos de alimentos, distribuídos por classe de despesa familiar, em salários minimos mensais (SM), São Paulo, 1975.

\begin{tabular}{|c|c|c|c|c|c|c|c|}
\hline \multirow{2}{*}{\multicolumn{2}{|c|}{$\begin{array}{l}\text { Grupo de } \\
\text { Alimentos }\end{array}$}} & \multicolumn{6}{|c|}{ Despesas mensais por familia (SM) } \\
\hline & & \multirow{2}{*}{$\begin{array}{c}\begin{array}{c}\text { Todas as } \\
\text { classes }\end{array} \\
861\end{array}$} & \multirow{2}{*}{$\frac{<1,0}{782}$} & \multirow{2}{*}{$\frac{\begin{array}{l}\geqslant 1,0 \\
<1,75\end{array}}{774}$} & \multirow{2}{*}{$\begin{array}{r}\geqslant 1,75 \\
<2,5\end{array}$} & \multirow{2}{*}{$\begin{array}{r}\geqslant 2,5 \\
<3,5 \\
966\end{array}$} & \multirow{2}{*}{$\begin{array}{l}\geqslant 3,5 \\
1.051\end{array}$} \\
\hline Cereais & W(kcal) & & & & & & \\
\hline & $\mathrm{Fe}(\mathrm{mg})$ & 3,5 & 3,2 & 3,1 & 3,7 & 3,9 & 4,3 \\
\hline \multirow{2}{*}{$\begin{array}{l}\text { Tubér- } \\
\text { culos }\end{array}$} & W(kcal) & 48 & 40 & 38 & 45 & 53 & 70 \\
\hline & $\mathrm{Fe}(\mathrm{mg})$ & 0,5 & 0,4 & 0,4 & 0.5 & 0,6 & 0,8 \\
\hline \multirow[t]{2}{*}{ Açúcar } & $\mathrm{W}(\mathrm{kcal})$ & $30: 3$ & 297 & 260 & 297 & 315 & 414 \\
\hline & $\mathrm{Fe}(\mathrm{mg})$ & 0,2 & 0,2 & 0,2 & 0.2 & 0,2 & 0,3 \\
\hline \multirow{2}{*}{$\begin{array}{l}\text { Legu- } \\
\text { minosas }\end{array}$} & $\mathrm{W}(\mathrm{kcal})$ & 198 & 240 & 200 & 204 & 202 & 206 \\
\hline & $\mathrm{Fe}(\mathrm{mg})$ & 4,3 & 5,4 & 4,5 & 4,6 & 4,5 & 4,6 \\
\hline \multirow{2}{*}{$\begin{array}{l}\text { Horta- } \\
\text { liças }\end{array}$} & W(kcal) & 28 & 17 & 18 & 24 & 30 & 47 \\
\hline & $\mathrm{Fe}(\mathrm{mg})$ & 0,9 & 0.5 & 0,6 & 0,7 & 1,0 & 1.5 \\
\hline \multirow[t]{2}{*}{ Frutas } & W(kcal) & 43 & 9 & 17 & 31 & 43 & 93 \\
\hline & $F e(m g)$ & 0.6 & 0,1 & 0,2 & 0,4 & 0,6 & 1.3 \\
\hline \multirow[t]{2}{*}{ Carnes } & W(kcal) & 184 & 73 & 104 & 165 & 206 & 320 \\
\hline & $\mathrm{Fe}(\mathrm{mg})$ & 2,4 & 0,9 & 1,4 & 2.1 & 2,7 & 4,2 \\
\hline \multirow[t]{2}{*}{ Ovos } & $\mathrm{W}(\mathrm{kcal})$ & 27 & 17 & 21 & 27 & 33 & 38 \\
\hline & $\mathrm{Fe}(\mathrm{mg})$ & 0,5 & 0,3 & 0,4 & 0,5 & 0,6 & 0,7 \\
\hline \multirow{2}{*}{$\begin{array}{l}\text { Leite e } \\
\text { deri- } \\
\text { vados }\end{array}$} & W(kcal) & 123 & 50 & 69 & 100 & 131 & 237 \\
\hline & $\mathrm{Fe}(\mathrm{mg})$ & 0,2 & 6.1 & 0.1 & 0,2 & 0,2 & 0,4 \\
\hline \multirow{3}{*}{$\begin{array}{l}\text { Gor- } \\
\text { duras }\end{array}$} & W(kcal) & 345 & 333 & 313 & 391 & 381 & 434 \\
\hline & $\mathrm{Fe}(\mathrm{mg})$ & 0 & 0 & 0 & 0 & 0 & 0 \\
\hline & W(kcal) & 2.155 & 1.858 & 1.814 & 2.159 & 2.360 & 2.910 \\
\hline \multirow[t]{2}{*}{ Total } & $\mathrm{Fe}(\mathrm{mg})$ & 13,1 & 11,1 & 10,9 & 12,9 & 14,3 & 18,1 \\
\hline & $\mathrm{dFe}$ & 6,1 & 6,0 & 6,0 & 6.0 & 6,1 & 6,2 \\
\hline
\end{tabular}

Fonte: Estimado a partir dos dados apresentados nas Tabelas $\mathbf{5 . 9 . 2}$ e $\mathbf{5 . 1 2 . 2}$ do Perfil estatístico de crianças e mães no Brasil (Fundação IBGE).

nutriente que deve ser acrescido ao alimento para adequá-lo às necessidades de consumo devido à forte dependència que a absorção do ferro dietético guarda com a qualidad. dos alimentos presentes na refeição. Como pode ser observado na Tabela 1 , embora o valor da $\mathrm{dFe}$ seja semelhante para as diversas classes de despesa familiar, o consumo de alimentos que melhoram $a$ absorção do ferro, como carnes e frutas ricas em ácido ascórbico, aumenta à medida etai que a família se insere em níveis mais elcvados de despesas correntes. Essa distri. buição de alimentos permite pressupor que a percentagem de absorção de ferro, entre as familias mais pobres, é menor.

Assim, um primeiro passo em direção à implantação de programa de fortificação com ferro é, obrigatoriamente, a identificação do potencial de absorção do ferro contido em refeiçōes habituais entre a população alvo, onde o alimento que se pretende fortificar esteja presente.

O objetivo deste trabalho foi o de quantificar a densidade do ferro biodisponivel ${ }^{5}$ 
SZARFARC, S.C. Densidade do ferro biodisponivel em uma dieta habitual no Estado de Såo Paulo.

Rev. Saúde públ., S. Paulo, 17:290-6, 1983.

(dFe B) nas refeições habituais entre os paulistas (desjejum, almoço e jantar), com vistas a contribuir no estabelecimento de bases para um programa de fortificação com ferro. A dFeB é definida com a quantidade de ferro (mg) absorvida por 1.000 kcal por indivíduos que absorvem $40 \%$ da dose, $\mathrm{Fe} /$ ascorbato, de referência ${ }^{5}$.

\section{METODOLOGIA}

\section{Refeições estudadas}

Os alimentos que compuseram as refeições estudadas foram estabelecidos com base nos inquéritos alimentares realizados pelo Departamento de Nutrição da Faculdade de Saúde Pública, Universidade de São Paulo *.

Eles foram assim constituidos:

Desjejum - pão $(55 \mathrm{~g})$, margarina $(8 \mathrm{~g})$, açúcar $(9 \mathrm{~g})$ e café $(50 \mathrm{ml}, 6 \%)$;

Almoço - arroz (59 g), feijão (44 g), carne $(56 \mathrm{~g})$, tomate $(9 \mathrm{~g})$ salsa $(1 \mathrm{~g})$, óleo $(20,4 \mathrm{~g})$, açúcar $(9 \mathrm{~g})$ e café $(50 \mathrm{ml}, 6 \%)$;

Jantar - arroz $(61 \mathrm{~g})$, feijão $(44 \mathrm{~g})$, ovo $(60 \mathrm{~g})$, cebola $(5,1 \mathrm{~g})$, óleo $(17,6)$, açúcar $(9 \mathrm{~g})$ e café $(50 \mathrm{ml}, 6 \%)$.

Os teores de energia e ferro das três refeiçōes foram:

Desjejum - $266 \mathrm{kcal}$ e $1,01 \mathrm{mg}$ de Fe não-heme.

Almoço - $723 \mathrm{kcal} 5,48 \mathrm{mg}$ de $\mathrm{Fe}$ não-heme e $0,9 \mathrm{mg}$ de $\mathrm{Fe}$ heme.

Jantar - $651 \mathrm{kcal}$ e $5,70 \mathrm{mg}$ de $\mathrm{Fe}$ não-heme.

\section{Amostra}

Participaram da pesquisa 28 voluntários, aparentemente sadios, com 18 anos ou mais. A determinação dos seus volumes sangüineos foi feita pela tabela de Tulane ${ }^{10}$ que leva em consideraçăo sexo, peso e idade.

\section{Absorção de ferro}

Para cada um dos individuos amostrados foram feitas 4 medidas de absorção de ferro ** sendo 3 para as refeições acima descritas e a $4^{a}$ medida para a absorção do ferro de uma dose-referência ingerida como uma solução de $3 \mathrm{mg}$ de ferro-ascorbato. A medida do ferro absorvido e do contido nas refeições foi feita pelo método de Dern e Hart 1,2 modificado.

A txperiência seguiu o modelo proposto por Layrisse e col.7, em 1974 e é descrita a seguir:

10 dia - ingestão do desjejum marcado com cerca de $0,6 \mu \mathrm{Ci}$ de ${ }^{59} \mathrm{Fe}$

20 dia - ingestão do almoço marcado com cerca de $2,0 \mu \mathrm{Ci}$ de ${ }^{\tilde{s}} \mathrm{Fe}$

$15^{\circ}$ dia - colheita de sangue para medidas de absorção do ferro ingerido nas 2 refeições

- ingestão do jantar marcado com cerca de $0,6 \mu \mathrm{Ci}$ de $59 \mathrm{Fe}$

$16^{\circ}$ dia - ingestão da dose-referência de $\mathrm{Fe}$-ascorbato marcado com cerca de $2,0 \mu \mathrm{Ci}$ de ${ }^{5} \mathrm{Fe}$

30. dia - colheita de sangue para medidas de absorção de ferro.

Os individuos amostrados foram orientados para chegarem, nos dias determinados para a pesquisa, em jejum e não consumirem qualquer alimento até 3 horas após a ingestão das refeições marcadas.

\section{RESULTADOS E COMENTARIOS}

Vários alimentos vêm sendo utilizados como veículo para nutrificação com ferro; - açúcar, selecionado, por nós, como o mais indicado para tal finalidade, apresenta algumas vantagens sobre outros alimentos.

\footnotetext{
* Grande proporģão de familias consomem refeições como as descritas, conforme observado entre os 1.310 inquéritos familiares realizados entre 1969-1972. Dados inéditos.

** Dosagens realizadas no Laboratório de Fisiopatologia do Instituto Venezolano de Investigaciones Cientificas.
} 
SZARFARC, S.C. Densidade do ferro blodisponivel em uma dieta habitual no Estado de São Paulo. Rev. Saide pribl., S. Paulo, 17:290-6, 1983.

T A B L A 2

Absorçăo de Fe em trés refeiçōes habituais entre a população do Estado de São Paulo e em dose-referência.

\begin{tabular}{|c|c|c|c|c|}
\hline Indentificação & $\begin{array}{c}\text { Dose-referência } \\
(55 \mathrm{Fe})\end{array}$ & $\begin{array}{l}\text { Desjejum } \\
(5 \theta \mathrm{Fe})\end{array}$ & $\begin{array}{l}\text { Almogo } \\
(55 \mathrm{Fe})\end{array}$ & $\begin{array}{l}\text { Jantar } \\
(50 \mathrm{Fe})\end{array}$ \\
\hline 1 & 27,9 & 0,3 & 2,3 & 3,0 \\
\hline 2 & 23,4 & 0.1 & 0,2 & 0,3 \\
\hline 3 & 49,3 & 4,8 & 6,0 & 8,7 \\
\hline 4 & 69,6 & 3.1 & 8,7 & 7,1 \\
\hline 5 & 42,5 & 7,2 & 11,5 & 4,5 \\
\hline 6 & 34,9 & 3.1 & 2.0 & 0.1 \\
\hline 7 & 21.2 & 2,3 & 0,4 & 2,4 \\
\hline 8 & 17,6 & 3,5 & 5,2 & 5,2 \\
\hline 9 & 1,4 & 2,7 & 1.3 & 0,0 \\
\hline 10 & 9,1 & 9,4 & 6,2 & 0,9 \\
\hline 11 & 36,8 & 18,3 & 15,4 & 2,5 \\
\hline 12 & 4,1 & 0,9 & 1,1 & 2,3 \\
\hline 13 & 7,0 & 2,7 & 5,3 & 0,9 \\
\hline 14 & 2,4 & 1,8 & 2,1 & 0,5 \\
\hline 15 & 39,9 & 6,4 & 3,6 & 3,5 \\
\hline 16 & 24,2 & 11,3 & 8,0 & 3,8 \\
\hline 17 & 13,0 & 0,6 & 0,5 & 0,6 \\
\hline 18 & 19,7 & 8,1 & 5,3 & 2,2 \\
\hline 19 & 12,6 & 4,1 & 1,6 & 0,6 \\
\hline 20 & 38,9 & 8,6 & 4,9 & 0,9 \\
\hline 21 & 57.6 & 6,0 & 2,6 & 4,4 \\
\hline 22 & 37,4 & 7.8 & 8,1 & 1,3 \\
\hline 23 & 12,1 & 0.7 & 0,1 & 1,1 \\
\hline 24 & 16.1 & 1,5 & 1.0 & 0,7 \\
\hline 25 & 43,7 & 3.2 & 0.7 & 1,8 \\
\hline 26 & 36,4 & 6,3 & 11,9 & 1,6 \\
\hline 27 & 0,9 & 1,1 & 2,7 & 0,1 \\
\hline 28 & 33.9 & 4,0 & 1,8 & 3,1 \\
\hline
\end{tabular}

Seu consumo é generalizado e a quantidade com que é ingerido é razoavelmente homogênea entre a população com até 3,5 salárics mínimos de despesa corrente (Tabela 1) e, principalmente, não contém substâncias que interfiram com a absorção do ferro ${ }^{6}$.

O açúcar é utilizado na alimentação sob várias formas e o resultado de sua fortificação com ferro varia dependendo da mistura onde ele esteja presente. Os refrigerantes, por exemplo, tem a absorção melhorada em cerca de $40 \%$ enquanto a mistura café-Jeite reduz a percentagem de aproveitamento do mineral e o suco de laranja triplica 0 mesmo ${ }^{6}$.
Nas três refeições estudadas, nas quais o açúcar participou como adoçante do café, foram obtidas as percentagens de absorção do ferro observadas na Tabela 2.

A absorção de ferro não hemínico de uma dada refeição e a absorçăo do ferro-ascorbato da dose referência, em diferentes individuos, guardam, uma relação linear entre si. Assim, é possivel obter através da inclinação da reta de regressão construida com essas duas medidas um indice de biodisponibilidade do ferro não heminico. Para torná-lo mais simples, Hallberg s propôs expressá-lo em função de um valor único de absorção da dose de referência - 40\% (valor que corresponde à absorção 
SZARFARC, S.C. Densidade do ferro blodisponíve! em uma dieta habitual no Estado de São Paulo. Rev. Saúde públ., S. Paulo, 17:290-6, 1983.

T A B E L A 3

Biodisponibilidade de ferro em três refeiçōes habituais no Estado de São Paulo.

\begin{tabular}{|c|c|c|c|c|c|c|c|c|}
\hline \multirow{2}{*}{ Refeição } & \multirow{2}{*}{$\begin{array}{c}\mathrm{w} \\
\mathrm{kcal}\end{array}$} & \multicolumn{2}{|c|}{ Ferro ingerido } & \multicolumn{4}{|c|}{ Ferro absorvido } & \multirow[b]{2}{*}{$D F e B$} \\
\hline & & $\begin{array}{l}\text { Não-heme } \\
(\mathrm{mg})\end{array}$ & $\begin{array}{c}\text { Heme } \\
(\mathrm{mg})\end{array}$ & $\begin{array}{l}\text { Não-heme } \\
(\%)\end{array}$ & $\begin{array}{l}\text { Heme } \\
(\%)\end{array}$ & $\begin{array}{l}\text { Não-heme } \\
\text { (mg) }\end{array}$ & $\begin{array}{r}\text { Heme } \\
(\mathrm{mg})\end{array}$ & \\
\hline Desjejum & 266 & 1,01 & - & 587 & - & 0,06 & - & 0,23 \\
\hline Almoço & 723 & 5,48 & 0,90 & 5,48 & 25.0 & 0,30 & 0,23 & 0.73 \\
\hline Jantar & 651 & 5,70 & - & 3.39 & - & 0.19 & - & 0,28 \\
\hline
\end{tabular}

de ferro da dose-referência em um individuo com deficiência de ferro-não anêmico).

Quanto mais severa a deficiência de ferro dos individuos, maior é a proporção de absorção do ferro ingerido. Assim, a fixação do valor $40 \%$ ć importante porque, caracterizando uma situação orgânica de depleção marcial máxima e concentração normal de hemoglobina, especifica as condiçōes fisiológicas correspondentes àquele indice de absorção de ferro referido.

Estimando os parâmetros para as retas de regressāo, onde $\mathrm{x}=$ absorção de ferro da dose-referência e $y=$ absorção de ferro da refeição, obtivemos os seguintes resultados:

Para o desjejum

$$
\begin{aligned}
& y=0,08 \cdot x+2,67 \\
& \text { Quando } x=40 \% \quad y=5,87 \%
\end{aligned}
$$

Para o almoço

$$
\begin{aligned}
& y=0,09 . x+1,88 \\
& \text { Quando } x=40 \% \quad y=5,48 \%
\end{aligned}
$$

Para o jantar

$$
\begin{aligned}
& y=0,08 \cdot x+0,19 \\
& \text { Quando } x=40 \% \quad y=3,39 \%
\end{aligned}
$$

A partir desses valores foi possivel calcular a densidade de ferro biodisponivel 5 (Tabela 3).

O valor nutritivo de uma refeição em relação a um nutriente é função da sua capacidade de suprir nas necessidades de cada grupo populacional; $0 \mathrm{dFeB}$; permite predizer esse valor para o ferro.

Como um exemplo desse poder do dFeB calculamos a capacidade de fornecimento de ferro da dieta estudada, para o homemtipo e mulher-tipo brasileiros ${ }^{8}$ pressuponc.n adecuação do consumo energético (distribuídos em $20 \%$ no desjejum e $40 \%$ no almoco e no jantar).

Considerando que a necessidade diária de absorção de ferro do homem é de $1 \mathrm{mg}$, a dieta estudada, consumida em quantidade adequada, estaria apta a satisfazê-la.

A dieta apresentada estima para a mulher a absorção de $1 \mathrm{mg}$ de ferro, valor bastante inferior ao necessário para atender à demanda de $90 \%$ das mulheres, $2,2 \mathrm{mg}{ }^{4}$.

Esses resultados sugerem que a fortificação do açúcar com ferro não é uma estratégia eficaz no combate à anemia, uma vez que é baixo o índice de biodisponibilidade de ferro (Tabela 4). Para obter um aumento do valor $\mathrm{dFeB}$ compativel com a necessidade da mulher seria preciso adicionar $5 \mathrm{mg}$ de ferro a cada refeição (Tabela 5).

Tal aumento de ferro redundaria em um consumo muito elevado do mineral entre outros grupos populacionais que, sem dúvida, ficariam predispostos à siderose. 
SZARFARC, S.C. Densidade do ferro biodisponível em uma dieta habitual no Estado de São Paulo. Rev. Saúde públ., S. Paulo, 1z:290-6, 1983.

T A B E L A 4

Estimativa da quantidade de ferro absorvida em três refeiçĩe:

\begin{tabular}{|c|c|c|c|c|c|c|}
\hline \multirow[t]{2}{*}{ Refeição } & \multirow[t]{2}{*}{ W (kcal) } & \multicolumn{2}{|c|}{$\begin{array}{c}\text { Homem-tipo } \\
F \in(m g)\end{array}$} & \multirow[t]{2}{*}{ W(kcal) } & \multicolumn{2}{|c|}{$\begin{array}{l}\text { Mulher-tipo } \\
\text { Fe(mg) }\end{array}$} \\
\hline & & Ingerido & Absorvido & & Ingerido & Absorvido \\
\hline Desjej::m & 580 & 2,20 & 0,13 & 430 & 1.63 & 0,10 \\
\hline Almoço & 1.160 & 10,23 & 0,85 & 860 & 7,59 & 0.63 \\
\hline Jantar & 1.160 & 10,16 & 0,34 & 860 & 7,53 & 0.25 \\
\hline Total & 2.900 & 22,59 & 1,32 & 2.150 & 16,75 & 0,98 \\
\hline
\end{tabular}

T A B E L A 5

Estimativa da quantidade de ferro absorvida, pela mulher-tipo após adição de $5 \mathrm{mg}$ de ferro a cada refeição.

\begin{tabular}{|c|c|c|c|}
\hline \multirow[t]{2}{*}{ Refeiça } & \multirow[t]{2}{*}{ W(kcal) } & \multicolumn{2}{|c|}{$\begin{array}{c}\text { Mulher-tipo } \\
\text { Fe(mg) }\end{array}$} \\
\hline & & Ingerido & Absorvido \\
\hline Desjej,m & 430 & 6.63 & 0.57 \\
\hline Almoço & 860 & 12.59 & 1,09 * \\
\hline Jantar & 860 & 12.53 & 0,48 \\
\hline Total & 2.150 & 31.75 & 2.14 \\
\hline
\end{tabular}

* $0.41 \mathrm{mg}$ deste total são de ferro heme.

É necessário, pois, tentar outras alternativas, que não a fortificação do açúcar, no combate à anemia ferropriva dada a baixa biodisponibilidade de ferro da dieta habitual entre a população do Estado de São Paulo.

\section{CONCLUSOES}

0 estudo da biodisponibilidade de ferro de uma dieta habitualmente consumida entre a população do Estado de São Paulo permitiu as seguintes conclusões:

- A densidade de ferro biodisponivel em refeição contendo pão, margarina e café adoçado com açúcar (266 kcal e $1,01 \mathrm{mg}$ Fe não-heme) é 0,23 ; da refeição contendo arroz + feijāo + carne e café com açúcar (723 kcal, $5,48 \mathrm{mg}$ Fe não- -heme e $0,9 \mathrm{mg} F e$ heme) é 0,$73 ; \mathrm{a}$ substituição de carne por ovo na refeição anterior (651 kcal e $5,70 \mathrm{mg} \mathrm{Fe}$ não-heme) diminui a biodisponibilidade do mineral para $\mathrm{dFeB}=0,28$.

- A dieta estudada, se consumida em quantidade adequada energeticamente, permite suprir a necessidade de ferro do homen.

- A dieta estudada, se consumida em quantidade que permita satisfazer a necessidade energética da mulher, não supre seu requerimento de ferro.

- Os resultados observados sugerem que, considerando o hábito alimentar, a fortificação do açúcar com ferro não é uma estratégia adequada no combate à anemia ferropriva. 
SZARFARC, S.C. Densidade do ferro biodisponível em uma dieta habitual no Estado de São Paulo, Rev. Saúde públ., S. Paulo, 17:290-6, 1983.

SZARFARC, S. C. [Bioavailable iron density in a customary dict in $\mathrm{S}$. Paulo State, Brazil]. Rev. Saúde públ., S. Paulo, 17:290-6, 1983.

ABSTRACT: A knowledge of the bioavailability of dietary iron is indis. pensable to the promotion of an iron fortification program. The quantification of that property was determined for three common meals, breakfast (bread, margarine, coffee with sugar), lunch (rice, beans, meat, coffee with sugar) and dinner (rice, beans, fried egg, coffee with sugar) served to 28 apparently healthy adults of both sexes. The bioavailable iron density $(\mathrm{dFe} B)$ found was: breakfast - $\mathrm{dFe} B=0,23$; lunch $-\mathrm{dFe} B=0,73$ and dinner $-\mathrm{dFe}$ $\mathbf{B}=0,28$. These results, insufficient to supply the iron required by women, suggest that fortification is not an effective strategy for control of iron deficiency anemia if applied to meals like those studied.

UNITERMS: Iron. Diet. Nutrition, surveys, S. Paulo State, Brazil.

\section{REFERENCIAS BIBLIOGRAFICAS}

1. DERN, R.J. \& HART W.L. Studies with doubly labeled iron. I - Simultaneous liquid scintilation counting of isotopos $\therefore \mathrm{Fe}$ and $39 \mathrm{Fe}$ as ferrous perchlorate. J. Lab. clin. Med., 57:322-30, 1961.

2. DERN. R.J. \& HART W.L. Studies with doubly labeled iron. II - Separation of iron from blood samples and preparations of ferrous perchlorate for liquid scintilation counting. $J$. Lab. clin. Med., 57:460-7, 1961.

3. FUNDAÇÃO IBGE. Perfil estatístico de crianças $e$ mães no Brasil. Rio de Janeiro, 1979

4. HALLBERG, L. Bioavailability of dietary iron in man. Ann. Rev. Nutr., 1:123-47, 1981.

5. HALLBERG, L. Bioatailable nutrient density: a new concept applied in the interpretation of food iron absorption data. Amer. $J$. clin. Nutr., 34:2.242-7, 1981.

6. INTERNATIONAL NUTRITIONAL ANEMIA CONSULTATIVE GROUP. Guidelines for the eradication of iron anemia: a report.
New York, the Nutrition Foundation Inc., 1977.

7. LAYRISSE, M. et al. Measurement of the total daily dietary iron absorption by the extrinsic tag model. Amer. J. clin. Nutr., 27:152-62, 1974.

8. MARTINS, I.S. Requerimentos de energia e nutrientes da população brasileira. Rev. Saúde públ., S. Paulo, 13 (supl. 1) :1-20, 1979 .

9. NATIONAL RESEARCH COUNCIL. Recom. mended dietary allowances. $9 \mathrm{th}$ ed Washington, D.C., 1980.

10. NADLER, S.B. et al. The Tulane table of blood volume in normal man. Survey. 51:224-32, 1962 .

11. WITTNER, A.J. et al. Nutrient density. evaluation of nutritional attributes of foods. J. Nutr. Educ., 9:26-30, 1977.

Recebido para publicasão em 20/12/1982 Aprovudo para publicaçao em 15/03/1983 